Monatsschr Kinderheilkd 2018 $\cdot 166: 998$ https://doi.org/10.1007/s00112-018-0586-z

(c) Springer Medizin Verlag GmbH, ein Teil von Springer Nature 2018

\section{Redaktion}

A. Borkhardt, Düsseldorf

S. Wirth, Wuppertal

CrossMark

\author{
N. Haiden ${ }^{1}$ für Ernährungskommission der Österreichischen Gesellschaft für \\ Kinder- und Jugendheilkunde e.V. (ÖGKJ) • B. Koletzko ${ }^{2}$ für \\ Ernährungskommission der Deutschen Gesellschaft für Kinder- und \\ Jugendmedizin e. V. (DGKJ) \\ ${ }^{1}$ Klinik für Klinische Pharmakologie, Medizinische Universität Wien, Wien, Österreich \\ ${ }^{2}$ Kinderklinik und Poliklinik im Dr. von Haunerschen Kinderspital, LMU - Ludwig-Maximilians-Universität, \\ München, Deutschland
}

\title{
Vegetarische und vegane Ernährung
}

Immer mehr Eltern haben den Wunsch, sich selbst und auch ihre Kinder mit unterschiedlichen vegetarischen Kostformen zu ernähren. Dies stellt Pädiater, die sowohl Mütter in der Stillzeit beraten als auch Säuglinge, Kleinkinder und größere Kinder in ihren Praxen betreuen, vor oft große Herausforderungen. Spezialwissen in Bezug auf einzelne Nährstoffe, deren Zufuhrmöglichkeiten, die suffiziente Supplementierung und Kontrolle von entsprechenden kindlichen Laborparametern sind hierfür nötig. Die deutsche wie auch die österreichische Ernährungskommission der jeweiligen Gesellschaften für Kinder- und Jugendmedizin haben sich voneinander unabhängig und auf unterschiedlich Weise mit der Thematik auseinandergesetzt und Positionspapiere als Hilfestellung für die pädiatrische Praxis erstellt. Die Stellungnahme der Deutschen Ernährungskommission gibt eine evidenzbasierte Literaturübersicht $\mathrm{zu}$ kritischen Nährstoffen bei vegetarischen Ernährungsformen. Die österreichische Stellungnahme, welche nur online verfügbar ist, gibt zusätzlich Normwerte für kritische Nährstoffe in Schwangerschaft, Stillzeit und Kleinkindalter an, sowie konkrete Empfehlungen, um die Nährstoffzufuhr bei vegetarischen Ernährungsformen zu beurteilen und ggf. zu verbessern. Pädiatern steht mit diesen beiden Papieren damit das Wissen zur Verfügung, das für die kompetente Betreuung betroffener Familien nötig ist.

\section{Korrespondenzadresse}

\section{Prof. Dr. B. Koletzko}

Kinderklinik und Poliklinik im Dr. von

Haunerschen Kinderspital, LMU - Ludwig-

Maximilians-Universität

Lindwurmstraße 4, 80337 München,

Deutschland

berthold.koletzko@med.uni-muenchen.de

Interessenkonflikt. N. Haiden und B. Koletzko geben an, dass kein Interessenkonflikt in Bezug auf den Inhalt dieses Editorials besteht. 